\title{
Automatic Scoring of Children's Read-Aloud Text Passages and Word Lists
}

\author{
Klaus Zechner and John Sabatini and Lei Chen \\ Educational Testing Service \\ Rosedale Road \\ Princeton, NJ 08541, USA \\ \{kzechner,jsabatini, 1 chen\}@ets.org
}

\begin{abstract}
Assessment of reading proficiency is typically done by asking subjects to read a text passage silently and then answer questions related to the text. An alternate approach, measuring reading-aloud proficiency, has been shown to correlate well with the aforementioned common method and is used as a paradigm in this paper.

We describe a system that is able to automatically score two types of children's read speech samples (text passages and word lists), using automatic speech recognition and the target criterion "correctly read words per minute". Its performance is dependent on the data type (passages vs. word lists) as well as on the relative difficulty of passages or words for individual readers. Pearson correlations with human assigned scores are around 0.86 for passages and around 0.80 for word lists.
\end{abstract}

\section{Introduction}

It has long been noted that a substantial number of U.S. students in the 10-14 years age group have deficiencies in their reading competence (National Center of Educational Statistics, 2006). With the enactment of the No Child Left Behind Act (2002), interest and focus on objectively assessing and improving this unsatisfactory situation has come to the forefront.
While assessment of reading is usually done posthoc with measures of reading comprehension, direct reading assessment is also often performed using a different method, oral (read-aloud) reading. In this paradigm, students read texts aloud and their proficiency in terms of speed, fluency, pronunciation, intonation etc. can be monitored directly while reading is in progress. In the reading research literature, oral reading has been one of the best diagnostic and predictive measures of foundational reading weaknesses and of overall reading ability (e.g., Deno et al., 2001; Wayman et al., 2007). An association between low reading comprehension and slow, inaccurate reading rate has been confirmed repeatedly in middle school populations (e.g., Deno \& Marsten, 2006). Correlations consistently fall in the $0.65-0.7$ range for predicting untimed passage reading comprehension test outcomes (Wayman et al., 2007).

In this paper, we investigate the feasibility of large-scale, automatic assessment of read-aloud speech of middle school students with a reasonable degree of accuracy (these students typically attend grades 6-8 and their age is in the 10-14 years range). If possible, this would improve the utility of oral reading as a large-scale, school-based assessment technique, making it more efficient by saving costs and time of human annotations and grading of reading errors.

The most widely used measure of oral reading proficiency is "correctly read words per minute" (cwpm) (Wayman et al., 2007). To obtain this measure, students' read speech samples are first 
recorded, then the reading time is determined, and finally a human rater has to listen to the recording and note all reading errors and sum them up. Reading errors are categorized into word substitutions, deletions etc.

We have several sets of digitally recorded readaloud samples from middle school students available which were not collected for use with automatic speech recognition (ASR) but which were scored by hand.

Our approach here is to pass the children's speech samples through an automatic speech recognizer and then to align its output word hypotheses with the original text that was read by the student. From this alignment and from the reading time, an estimate for the above mentioned measure of cwpm can then be computed. If the automatically computed cwpm measures are close enough to those obtained by human hand-scoring, this process may be employed in real world settings eventually to save much time and money.

Recognizing children's speech, however, has been shown to be substantially harder than adult speech (Lee et al., 1999; Li and Russell, 2002), which is partly due to children's higher degree of variability in different dimensions of language such as pronunciation or grammar. In our data, there was also a substantial number of non-native speakers of English, presenting additional challenges. We used targeted training and adaptation of our ASR systems to achieve reasonable word accuracies. While for text passages, the word accuracy on unseen speakers was about $72 \%$, it was only about $50 \%$ for word lists, which was due in part to a higher percentage of non-native speakers in this data set, to the fact that various sources of noise often prevented the recognizer from correctly locating the spoken words in the signal, and also due to our choice of a uniform language model since conventional n-gram models did not work on this data with many silences and noises between words.

The remainder of this paper is organized as follows: in Section 2 we review related work, followed by a description of our data in Section 3. Section 4 provides a brief description of our speech recognizer as well as the experimental setup. Section 5 provides the results of our experiments, followed by a discussion in Section 6 and conclusions and future work in Section 7.

\section{Related work}

Following the seminal paper about the LISTEN project (Mostow et al. 1994), a number of studies have been conducted on using automatic speech recognition technology to score children's read speech.

Similar to automated assessment of adults' speech (Neumeyer, Franco et al. 2000; Witt, 1999), the likelihood computed in the Hidden Markov Model (HMM) decoding and some measurements of fluency, e.g., speaking rate, are widely used as features for predicting children's speaking proficiency. Children's speech is different than adults'. For example, children's speech exhibits higher fundamental frequencies (F0) than adults on average. Also, children's more limited knowledge of vocabulary and grammar results in more errors when reading printed text. Therefore, to achieve high-quality recognition on children's speech, modifications have to be made on recognizers that otherwise work well for adults.

In the LISTEN project (Mostow et al., 1994), the basic technology is to use speech recognition to classify each word of text as correctly read or not. Such a classification task is hard in that the children's speaking deviations from the text may include arbitrary words and non-words. In a study, they modeled variations by the modification of the lexicon and the language model of the Sphinx ${ }^{1}$ speech recognizer.

Recently, the Technology Based Assessment of Language and Literacy project (TBALL, (Alwan, 2007)) has been attempting to assess and evaluate the language and literacy skills of young children automatically. In the TBALL project, a variety of tests including word verification, syllable blending, letter naming, and reading comprehension, are jointly used. Word verification is an assessment that measures the child's pronunciation of readaloud target words. A traditional pronunciation verification method based on log-likelihoods from HMM models is used initially (Tepperman et al., 2006). Then an improvement based on a Bayesian network classifier (Tepperman et al., 2007) is em-

\footnotetext{
${ }^{1}$ See http://cmusphinx.sourceforge.net/html/cmusphinx.php
} 
ployed to handle complicated errors such as pronunciation variations and other reading mistakes.

Many other approaches have been developed to further improve recognition performance on children's speech. For example, one highly accurate recognizer of children's speech has been developed by Hagen et al. (2007). Vocal tract length normalization (VTLN) has been utilized to cope with the children's different acoustic properties. Some special processing techniques, e.g., using a general garbage model to model all miscues in speaking, have been devised to improve the language model used in the recognition of children's speech ( $\mathrm{Li}$ et al., 2007).

\section{Data}

For both system training and evaluation, we use a data set containing 3 passages read by the same 265 speakers (Set1) and a fourth passage (a longer version of Passage 1), read by a different set of 55 speakers (Set2). Further, we have word lists read by about 500 different speakers (Set3). All speakers from Set $1^{2}$ and most (84\%) from the third set were U. S. middle school students in grades 6-8 (age 10-14). A smaller number of older students in grades 10-12 (age 15-18) was also included in the third set (16\%). ${ }^{34}$

In terms of native language, about $15 \%$ of Set 1 and about $76 \%$ of Set $^{5}$ are non-native speakers of English or list a language different from English as their preferred language.

Table 1 provides the details of these data sets. In the word lists data set, there are 178 different word lists containing 212 different word types in total (some word lists were read by several different students).

All data was manually transcribed using a spreadsheet where each word is presented in one line and the annotator, who listens to the audio file, has to

\footnotetext{
${ }^{2}$ For Set1, we have demographics for 254 of 265 speakers (both for grade level and native language).

${ }^{3}$ Grade demographics are available for 477 speakers of Set3.

${ }^{4}$ We do not have demographic data for the small Set2 (55 speakers).

${ }^{5}$ This set (Set 3) has information on native language for 165 speakers.
}

mark-up any insertions, substitutions or deletions by the student.

\begin{tabular}{|l|l|l|}
\hline Name & Recordings & $\begin{array}{l}\text { Length in } \\
\text { words }\end{array}$ \\
\hline $\begin{array}{l}\text { Passage 1 } \\
\text { (“Bed”, Set1-A) }\end{array}$ & 265 & 158 \\
\hline $\begin{array}{l}\text { Passage 2 } \\
\text { (“Girls”, Set1-B) }\end{array}$ & 265 & 74 \\
\hline $\begin{array}{l}\text { Passage 3 } \\
\text { (“Keen”, Set1-C) }\end{array}$ & 265 & 100 \\
\hline $\begin{array}{l}\text { Passage 4 } \\
\text { (“Bed*”) (Set2) }\end{array}$ & 55 & 197 \\
\hline Word lists (Set3) & 590 & 62 (average) \\
\hline
\end{tabular}

Table 1. Text passages and word lists data sets.

For ASR system training only, we additionally used parts of the OGI (Oregon Graduate Institute) and CMU (Carnegie Mellon University) Kids data sets as well (CSLU, 2008; LDC, 1997).

\section{ASR system and experiments}

The ASR system's acoustic model (AM) was trained using portions of the OGI and CMU Kids' corpora as well as a randomly selected sub-set of our own passage and word list data sets described in the previous section. About $90 \%$ of each data set (Set1, Set2, Set3) was used for that purpose. Since the size of our own data set was too small for AM training, we had to augment it with the two mentioned corpora (OGI, CMU Kids), although they were not a perfect match in age range and accent.

All recordings were first converted and downsampled to $11 \mathrm{kHz}$, mono, 16 bit resolution, PCM format. There was no speaker overlap between training and test sets.

For the language model (LM), two different models were created: for passages, we built an interpolated trigram LM where $90 \%$ of the weight is assigned to a LM trained only on the 4 passages from the training set (Set1, Set2) and $10 \%$ to a generic LM using the Linguistic Data Consortium (LDC) Broadcast News corpus (LDC, 1997). The dictionary contains all words from the transcribed passages in the training set, augmented with the 1,000 most frequent words from the Broadcast News corpus. That way, the LM is not too restrictive and allows the recognizer to hypothesize some 
reading mistakes not already encountered in the human transcriptions of the training set.

For the word lists, a trigram LM was found to be not working well since the words were spoken in tween and automatic removal of these silences proved too hard given other confounding factors such as microphone, speaker, or background noise. Therefore it was decided to implement a grammar LM for the word list decoder where all possible words are present in a network that allows them to occur at any time and in any sequence, allowing for silence and/or noises in between words. This model with uniform priors, however, has the disin the word list training set, such as common mispronunciations and is therefore more restrictive than the LM for text passages.

One could make the argument of using forced alignment instead of a statistical LM to determine reading errors. In fact, this approach is typically used when assessing the pronunciation of read speech. However, in our case, the interest is more in determining how many words were read correctly in the sequence of the text (and how fast they were read) as opposed to details in pronunciation. Further, even if we had confidence scores attached to words in forced alignment, deciding on which of the words obtained low confidence due to poor pronunciation or due to substitution would not be an easy decision. Finally, word deletions and insertions, if too frequent, might prevent the forced alignment algorithm from terminating.

After training was complete, we tested the recognizer on the held-out passage and word list data. After recognizing, we computed our target measure of "correct words per minute" (cwpm) according to the following formula ( $\mathrm{W}=$ all words in a text, $\mathrm{S}=$ substitutions, $\mathrm{D}=$ deletions, $\mathrm{T}=$ reading time in minutes), performing a string alignment between the recognizer hypothesis and the passage or word list to be read:

$$
c w p m=\frac{W-S-D}{T}
$$
isolation with sometimes significant pauses in beadvantage of not including any words not present

time and therefore can be considered to be accounted for already in the formula.

Next, we performed an experiment that looks at whether automatic scoring of read-aloud speech allows for accurate predictions of student placements in broad cohorts of reading proficiency.

We then also look more closely at typical errors made by human readers and the speech recognizer. All these experiments are described and discussed in the following section.

Table 2 describes the set-up of the experiments. Note that Passage4 (Set2) was included only in the training but not in the evaluation set since this set was very small. As mentioned in the previous section, most speakers from the passage sets read more than one passage and a few speakers from the word lists set read more than one word list.

\begin{tabular}{|l|l|l|l|}
\hline Data set & Recordings & Speakers & $\begin{array}{l}\text { Language } \\
\text { model } \\
\text { type }\end{array}$ \\
\hline $\begin{array}{l}\text { Passages1- } \\
3\end{array}$ & 101 & 37 & Trigram \\
\hline Word lists & 42 & 38 & Grammar \\
\hline
\end{tabular}

Table 2. Experiment set-up (evaluation sets).

\section{Results}

\subsection{Overall results}

Table 3 depicts the results of our evaluation run with the ASR system described above. Word accuracy is measured against the transcribed speaker reference (not against the true text that was read). Word accuracy is computed according to Equation (2), giving equal weight to reference and ASR hypothesis (c=correct, s=substitutions, d=deletions, $\mathrm{i}=$ insertions). This way, the formula is unbiased with respect to insertions or deletions:

The reason that insertions are not considered here is that they contribute to an increase in reading 


\begin{tabular}{|l|l|l|l|l|l|}
\hline Data set & Recordings & Speakers & $\begin{array}{l}\text { Average word } \\
\text { Accuracy over all } \\
\text { speech sample }\end{array}$ & $\begin{array}{l}\text { Minimum word } \\
\text { accuracy on a } \\
\text { speech sample }\end{array}$ & $\begin{array}{l}\text { Maximum word } \\
\text { accuracy on a speech } \\
\text { sample }\end{array}$ \\
\hline $\begin{array}{l}\text { All Passages } \\
\text { (1-3) }\end{array}$ & 101 & 37 & 72.2 & 20.4 & 93.8 \\
\hline $\begin{array}{l}\text { Passage1 } \\
\text { (“Bed”) }\end{array}$ & 28 & 28 & 70.8 & 20.4 & 83.6 \\
\hline $\begin{array}{l}\text { Passage2 } \\
\text { (“Girls”) }\end{array}$ & 36 & 36 & 64.1 & 25.4 & 85.7 \\
\hline $\begin{array}{l}\text { Passage3 } \\
\text { (“Keen”) }\end{array}$ & 37 & 37 & 77.7 & 27.4 & 93.8 \\
\hline Word lists & 42 & 38 & 49.6 & 10.8 & 78.9 \\
\hline
\end{tabular}

Table 3. ASR experiment results (word accuracies in percent)

The typical run-time on a $3.2 \mathrm{GHz}$ Pentium processor was less than 30 seconds for a recording (faster than real time).

We next compute cwpm measures for both human annotations (transcripts, "gold standard") and machine (ASR) hypotheses

Human annotators went over each read passage and word list and marked all reading errors of the speakers (here, only deletions and substitutions are relevant). The reading time is computed directly from the speech sample, so machine and human cwpm scores only differ in error counts of deletions and substitutions. Currently we only have one human annotation available per speech sample, but we aim to obtain a second annotation for the purpose of determining inter-annotator agreement.

Table 4 presents the overall results of comparing machine and human cwpm scoring. We performed both Pearson correlation as well as Spearman rank correlation. While the former provides a more generic measure of cwpm correlation, the latter focuses more on the question of the relative performance of different speakers compared to their peers which is usually the more interesting question in practical applications of reading assessment. Note that unlike for Table 3, the ASR hypotheses are now aligned with the text to be read since in a real-world application, no human transcriptions would be available.

We can see that despite the less than perfect recognition rate of the ASR system which causes a much lower average estimate for cwpm or cw (for wordlists), both Pearson and Spearman correlation coefficients are quite high, all above 0.7 for Spearman rank correlation and equal to 0.8 or higher for the Pearson product moment correlation. This is encouraging as it indicates that while current ASR technology is not yet able to exactly transcribe children's read speech, it is

\begin{tabular}{|l|l|l|l|l|}
\hline Data set & $\begin{array}{l}\text { Gold } \\
\text { cwpm }\end{array}$ & $\begin{array}{l}\text { ASR- } \\
\text { based } \\
\text { cwpm }\end{array}$ & $\begin{array}{l}\text { Pearson } \\
\text { r corre- } \\
\text { lation }\end{array}$ & $\begin{array}{l}\text { Spearman } \\
\text { rank cor- } \\
\text { relation }\end{array}$ \\
\hline $\begin{array}{l}\text { All Pas- } \\
\text { sages } \\
\text { (1-3) }\end{array}$ & 152.0 & 109.8 & 0.86 & NA \\
\hline $\begin{array}{l}\text { Passage1 } \\
\text { (Bed) }\end{array}$ & 174.3 & 123.5 & 0.87 & 0.72 \\
\hline $\begin{array}{l}\text { Passage2 } \\
\text { (Girls) }\end{array}$ & 133.1 & 86.5 & 0.86 & 0.73 \\
\hline $\begin{array}{l}\text { Passage3 } \\
\text { (Keen) }\end{array}$ & 153.4 & 122.2 & 0.86 & 0.77 \\
\hline $\begin{array}{l}\text { Word } \\
\text { lists* }\end{array}$ & 48.0 & 29.4 & 0.80 & 0.81 \\
\hline
\end{tabular}

Table 4. CWPM results for passages and word lists. All correlations are significant at $\mathrm{p}<0.01$. *For word lists, we use "cw" (correct words, numerator of Equation (1)) as the measure, since students were not told to be rewarded for faster reading time here.

possible to use its output to compute reasonable read-aloud performance measures such as cwpm 
which can help to quickly and automatically assess reading proficiencies of students.

\subsection{Cohort assignment experiment}

To follow up on the encouraging results with basic and rank correlation, we conducted an experiment to explore the question of practical importance whether the automatic system can assign students to reading proficiency cohorts automatically.

For better comparison, we selected those 27 students from 37 total who read all 3 passages (Set 1) and grouped them into three cohorts of 9 students each, based on their human generated cwpm score for all passages combined: (a) proficient (cwpm>190), (b) intermediate $(135<\mathrm{cwpm}<190)$, and (c) low proficient $(\mathrm{cwpm}<135)$.

We then had the automatic system predict each student's cohort based on the cwpm computed from ASR. Since ASR-based cwpm values are consistently lower than human annotator based cwpm values, the automatic cohort assignment is not based on the cwpm values but rather on their ranking.

The outcome of this experiment is very encouraging in that there were no cohort prediction errors by the automatic system. While the precise ranking differs, the system is very well able to predict overall cohort placement of students based on cwpm.

\subsection{Overall comparison of students' reading er- rors and ASR recognition errors}

To look into more detail of what types of reading errors children make and to what extent they are reflected by the ASR system output, we used the sclite-tool by the National Institute for Standards and Technology (NIST, 2008) and performed two alignments on the evaluation set:

1. TRANS-TRUE: Alignment between human transcription and true passage or word list text to be read: this alignment informs us about the kinds of reading errors made by the students.

2. HYPO-TRANS: Alignment between the ASR hypotheses and the human transcriptions; this alignment informs us of ASR errors. (Note that this is different from the experiments reported in Table 4 above where we aligned the ASR hypotheses with the true reference texts to compute cwpm.)
Table 5 provides general statistics on these two alignments.

\begin{tabular}{|l|l|l|l|l|}
\hline Data set & Alignment & SUB & DEL & INS \\
\hline $\begin{array}{l}\text { Passages } \\
1-3\end{array}$ & $\begin{array}{l}\text { TRANS- } \\
\text { TRUE }\end{array}$ & $2.0 \%$ & $6.1 \%$ & $1.8 \%$ \\
\hline $\begin{array}{l}\text { Pas- } \\
\text { sages1-3 }\end{array}$ & $\begin{array}{l}\text { HYPO- } \\
\text { TRANS }\end{array}$ & $18.7 \%$ & $9.6 \%$ & $8.1 \%$ \\
\hline $\begin{array}{l}\text { Word } \\
\text { lists }\end{array}$ & $\begin{array}{l}\text { TRANS- } \\
\text { TRUE }\end{array}$ & $5.6 \%$ & $6.2 \%$ & $0.6 \%$ \\
\hline $\begin{array}{l}\text { Word } \\
\text { lists }\end{array}$ & $\begin{array}{l}\text { HYPO- } \\
\text { TRANS }\end{array}$ & $42.0 \%$ & $8.9 \%$ & $6.4 \%$ \\
\hline
\end{tabular}

Table 5. Word error statistics on TRANS-TRUE

and HYPO-TRANS alignments for both evaluation data sets.

From Table 5 we can see that while for students, deletions occur more frequently than substitutions and, in particular, insertions, the ASR system, due to its imperfect recognition, generates mostly substitutions, in particular for the word lists where the word accuracy is only around $50 \%$.

Further, we observe that the students' average reading word error rate (only taking into account substitutions and deletions as we did above for the cwpm and cw measures) lies around $8 \%$ for passages and $12 \%$ for wordlists (all measured on the held-out evaluation data).

\subsection{Specific examples}

Next, we look at some examples of frequent confusion pairs for those 4 combinations of data sets and alignments. Table 6 lists the top 5 most frequent confusion pairs (i.e., substitutions).

For passages, all of the most frequent reading errors by students are morphological variants of the target words, whereas this is only true for some of the ASR errors, while other ASR errors can be far off the target words. For word lists, student errors are sometimes just orthographically related to the target word (e.g., "liner" instead of "linear"), and sometimes of different part-of-speech (e.g., "equally" instead of "equality"). ASR errors are typically related to the target word by some phonetic similarity (e.g., "example” instead of "simple”). 
Finally, we look at a comparison between errors made by the students and the fraction of those correctly identified by the ASR system in the recognition hypotheses. Table 7 provides the statistics on these matched errors for text passages and word lists.

\begin{tabular}{|c|c|c|c|c|}
\hline $\begin{array}{l}\text { Data } \\
\text { set }\end{array}$ & $\begin{array}{l}\text { Align- } \\
\text { ment }\end{array}$ & $\begin{array}{l}\text { Refer- } \\
\text { ence }\end{array}$ & $\begin{array}{l}\text { Spoken/ } \\
\text { recog- } \\
\text { nized }\end{array}$ & Count \\
\hline $\begin{array}{l}\text { Pas- } \\
\text { sages } \\
1-3\end{array}$ & $\begin{array}{l}\text { TRANS } \\
\text {-TRUE }\end{array}$ & $\begin{array}{l}\text { asks } \\
\text { savings } \\
\text { projects } \\
\text { teacher's } \\
\text { time }\end{array}$ & $\begin{array}{l}\text { ask } \\
\text { saving } \\
\text { project } \\
\text { teacher } \\
\text { times } \\
\end{array}$ & $\begin{array}{l}6 \\
5 \\
4 \\
4 \\
4\end{array}$ \\
\hline $\begin{array}{l}\text { Pas- } \\
\text { sages } \\
1-3\end{array}$ & $\begin{array}{l}\text { HYPO- } \\
\text { TRANS }\end{array}$ & $\begin{array}{l}\text { storm } \\
\text { lee's } \\
\text { lee's } \\
\text { observer } \\
\text { thousand }\end{array}$ & $\begin{array}{l}\text { storms } \\
\text { be } \\
\text { we } \\
\text { and } \\
\text { the }\end{array}$ & $\begin{array}{l}11 \\
6 \\
6 \\
6 \\
6 \\
\end{array}$ \\
\hline $\begin{array}{l}\text { Word } \\
\text { lists }\end{array}$ & $\begin{array}{l}\text { TRANS } \\
\text {-TRUE }\end{array}$ & $\begin{array}{l}\text { nature } \\
\text { over- } \\
\text { sleep } \\
\text { equality } \\
\text { linear } \\
\text { ware- } \\
\text { housed } \\
\end{array}$ & $\begin{array}{l}\begin{array}{l}\text { Natural } \\
\text { overslept }\end{array} \\
\text { equally } \\
\text { liner } \\
\text { ware- } \\
\text { house }\end{array}$ & $\begin{array}{l}6 \\
5 \\
4 \\
4 \\
3\end{array}$ \\
\hline $\begin{array}{l}\text { Word } \\
\text { lists }\end{array}$ & $\begin{array}{l}\text { HYPO- } \\
\text { TRANS }\end{array}$ & $\begin{array}{l}\text { plan } \\
\text { see } \\
\text { simple } \\
\text { unoffi- } \\
\text { cial } \\
\text { loud }\end{array}$ & $\begin{array}{l}\text { planned } \\
\text { season } \\
\text { example } \\
\text { competi- } \\
\text { tion } \\
\text { through- } \\
\text { out }\end{array}$ & $\begin{array}{l}8 \\
6 \\
6 \\
5 \\
4\end{array}$ \\
\hline
\end{tabular}

Table 6. Top 5 most frequent confusion pairs for passages and word list evaluation sets in two different alignments. For passages, substitutions among closed class words such as determiners or prepositions are omitted.

Table 7 shows that while for text passages, almost half of the relevant errors (substitutions and deletions) were correctly identified by the recognizer, for word lists, this percentage is substantially smaller.

\section{Discussion}

The goal of this paper is to evaluate the possibility of creating a system for automatic oral reading assessment for middle school children, based on text passages and word lists.

We decided to use the common reading proficiency measure of "correct words per minute" which enables us to align ASR word hypotheses with the correct texts, estimate cwpm based on this alignment and the reading time, and then compare the automatically estimated cwpm with human annotations of the same texts.

\begin{tabular}{|l|l|}
\hline Data set / error type & $\begin{array}{l}\text { Percentage of correctly } \\
\text { identified errors }\end{array}$ \\
\hline Passages 1-3 - SUB & 20.6 \\
\hline Passages 1-3 - DEL & 56.4 \\
\hline $\begin{array}{l}\text { Passages 1-3 - } \\
\text { SUB+DEL }\end{array}$ & 47.7 \\
\hline Word lists - SUB & 2.7 \\
\hline Word lists - DEL & 29.4 \\
\hline $\begin{array}{l}\text { Word lists - } \\
\text { SUB+DEL }\end{array}$ & 16.8 \\
\hline
\end{tabular}

Table 7. Statistics on matched errors: percentage of students' reading errors (substitutions and deletions) that were also correctly identified by the ASR system.

We built a recognizer with an acoustic model based on CMU and OGI kids' corpora as well as about $90 \%$ of our own text passages and word list data (Sets 1-3). For the in-context reading (text passages) we trained a trigram model focused mostly on transcriptions of the passages. For the out-of-context isolated word reading, we used a grammar language model where every possible word of the word lists in the training set can follow any other word at any time, with silence and/or noise between words. (While this was not our preferred choice, standard n-gram language models performed very poorly given the difficulty of removing inter-word silences or noise automatically.)

Given how hard ASR for children's speech is and given our small matched data sets, the word accuracy of $72 \%$ for text passages was not unreasonable and was acceptable, particularly in a first development cycle. The word accuracy of only about $50 \%$ for word lists, however, is more prob- 
lematic and we conjecture that the two main reasons for the worse performance were (a) the absence of time stamps for the location of words which made it sometimes hard for the recognizer to locate the correct segment in the signal for word decoding (given noises in between), and (b) the sometimes poor recording conditions where volumes were set too high or too low, too much background or speaker noise was present etc. Further, the high relative number of non-native speakers in that data set may also have contributed to the lower word accuracy of the word lists.

While the current data collection had not been done with speech recognition in mind, in future data collection efforts, we will make sure that the sound quality of recordings is better monitored, with some initial calibration, and that we store time stamps when words are presented on the screen to facilitate the recognition task and to allow the recognizer to expect one particular word at one particular point in time.

Despite imperfect word accuracies, however, for both passages and word lists we found encouragingly high correlations between human and automatic cwpm measures (cw measures for word lists). Obviously, the absolute values of cwpm differ greatly as the ASR system generates many more errors on average than the readers, but both Pearson correlation as well as Spearman rank correlation measures are all above 0.7 . This means that if we would use our automatic scoring results to rank students' reading proficiency, the ranking order would be overall quite similar to an order produced by human annotators. This observation about the rank, rather than the absolute value of cwpm, is important in so far as it is often the case that educators are interested in separating "cohorts" of readers with similar proficiency and in particular to identify the lowest performing cohort for additional reading practice and tutoring.

An experiment testing the ability of the system to place students into three reading proficiency cohorts based on cwpm was very encouraging in that all 27 students of the test set were placed in the correct cohort by the system.

When we compare frequent student errors with those made by the machine (Table 6), we see that often times, students just substitute slight morphological variants (e.g., "ask” for "asks”), whereas in the ASR system, errors are typically more complex than just simple substitutions of morphological variants. However, in the case of word lists, we do find substitutions with related phonological content in the ASR output (e.g., "example” for "simple”). Finally, we observed that, only for the text passages, the ASR system could correctly identify a substantial percentage of readers' substitutions and deletions (about 48\%, see Table 7). This is also encouraging as it is a first step towards meaningful feedback in a potential interactive setting. However, we here only look at recall - because of the much larger number of ASR substitutions, precision is much lower and therefore the risk of overcorrection (false alarms) is still quite high.

Despite all of the current shortcomings, we feel that we were able to demonstrate a "proof-ofconcept" with our initial system in that we can use our trained ASR system to make reliable estimates on students' reading proficiency as measured with "correct words per minute", where correlations between human and machine scores are in the 0.80-0.86 range for text passages and word lists.

\section{Conclusions and future work}

This paper demonstrates the feasibility of building an automatic scoring system for middle school students' reading proficiency, using a targeted trained speech recognition system and the widely used measure of "correctly read words per minute" (cwpm).

The speech recognizer was trained both on external data (OGI and CMU kids' corpora) and internal data (text passages and word lists), yielding two different modes for text passages (trigram language model) and word lists (grammar language model). Automatically estimated cwpm measures agreed closely with human cwpm measures, achieving 0.8 and higher correlation with Pearson and 0.7 and higher correlation with Spearman rank correlation measures.

Future work includes an improved set-up for recordings such as initial calibration and on-line sound quality monitoring, adding time stamps to recordings of word lists, adding more data for training/adaptation of the ASR system, and exploring other features (such as fluency features) and their potential role in cwpm prediction. 


\section{Acknowledgements}

The authors would like to acknowledge the contributions of Kathy Sheehan, Tenaha O'Reilly and Kelly Bruce to this work. We further are grateful for the useful feedback and suggestions from our colleagues at ETS and the anonymous reviewers that greatly helped improve our paper.

\section{References}

Alwan, A. (2007). A System for Technology Based Assessment of Language and Literacy in Young Children: the Role of Multiple Information Sources. Proceedings of MMSP, Greece.

Center for Spoken Language Understanding (CSLU), 2008. Kids' Speech Corpus, http://www.cslu.ogi.edu/corpora/kids/.LDC, BN.

Deno, S. L., Fuchs, L. S., Marston, D., \& Shin, J. (2001). Using curriculum-based measurements to establish growth standards for students with learning disabilities. School Psychology Review, 30(4), 507-524.

Deno, S. L. and D. Marsten (2006). Curriculumbased measurement of oral reading: An indicator of growth in fluency. What Research Has to Say about Fluency Instruction. S. J. Samuels and A. E. Farstrup. Newark, DE, International Reading Association: 179-203.

Hagen, A., B. Pellom, \& R. Cole. (2007). "Highly accurate children's speech recognition for interactive reading tutors using subword units." Speech Communication 49(6): 861-873.

Lee, S., A. Potamianos, \& S. Narayanan. (1999). "Acoustics of children's speech: developmental changes of temporal and spectral parameters." Journal of Acoustics Society of American (JASA) 105: 1455-1468.

Li, X., Y. C. Ju, L. Deng \& A. Acero. (2007). Efficient and Robust Language Modeling in an Automatic Children's Reading Tutor System. Proc. IEEE International Conference on Acoustics, Speech and Signal Processing ICASSP 2007.
Li, Q. and M. Russell (2002). An analysis of the causes of increased error rates in children's speech recognition. ICSLP. Denver, CO.

Linguistic Data Consortium (LDC), 1997. 1996 English Broadcast News Speech (HUB4), LDC97S44.

Linguistic Data Consortium (LDC), 1997. The CMU Kids Corpus, LDC97S63.

Mostow, J., S. F. Roth, G. Hauptmann \& M. Kane. (1994). A prototype reading coach that listens. AAAI '94: Proceedings of the twelfth national conference on Artificial intelligence, Menlo Park, CA, USA, American Association for Artificial Intelligence.

National Center of Educational Statistics. (2006). National Assessment of Educational Progress. Washington DC: U.S. Government Printing Office.

National Institute for Standards and Technology (NIST), 2008. Sclite software package. http://www.nist.gov/speech/tools/

Neumeyer, L., H. Franco, V. Digalakis \& M. Weintraub. (2000). "Automatic Scoring of Pronunciation Quality." Speech Communication 6.

No Child Left Behind Act of 2001, Pub. L. No. 107-110, 115 Stat. 1425 (2002).

Tepperman, J., J. Silva, A. Kazemzadeh, H. You, S. Lee, A. Alwan \& S. Narayanan. (2006). Pronunciation verification of children's speech for automatic literacy assessment. INTERSPEECH2006. Pittsburg, PA.

Tepperman, J., M. Black, P. Price, S. Lee, A. Kazemzadeh, M. Gerosa, M. Heritage, A. Alwan \& S. Narayanan.(2007). A bayesian network classifier for word-level reading assessment. Proceedings of ICSLP, Antwerp, Belgium.

Wayman, M. M., Wallace, T., Wiley, H. I., Ticha, R., \& Espin, C. A. (2007). Literature synthesis on curriculum-based measurement in reading. The Journal of Special Education, 41(2), 85-120.

Witt, S. M. (1999). Use of Speech Recognition in Computer-assisted Language Learning, University of Cambridge. 